\title{
A novel monoclonal antibody against the von Willebrand Factor A2 domain reduces its cleavage by ADAMTS13
}

\author{
Lulu Zhang ${ }^{1,2}$, Jian Su ${ }^{1,2}$, Fei Shen ${ }^{1,2}$, Zhenni Ma ${ }^{1,2}$, Yiming Zhao ${ }^{1,2}$, Lijun Xia ${ }^{1,2}$ and Changgeng Ruan ${ }^{1,2^{*}}$ (D)
}

\begin{abstract}
We developed a novel murine monoclonal antibody (mAb) against the C-terminal a-helix of the human von Willebrand factor A2, designated SZ-179. We showed that SZ-179 inhibited the interactions between VWF and ADAMTS13 and prevented the degradation of high molecular weight WWF multimers. Importantly, SZ-179 reduced the proteolysis of WWF-R1597W mutant by rADAMTS13 dose-dependently under native conditions. Our findings reveal a potential therapeutic target for bleeding disorders.
\end{abstract}

Keywords: von Willebrand factor, Monoclonal antibody, ADAMTS13

\section{Findings}

ADAMTS13 (a disintegrin and metalloproteinase with a thrombospondin type 1 motif, member 13 ) regulates the multimeric size of von Willebrand factor (VWF) by cleaving the Tyr1605-Met1606 bond in the VWF A2 domain (VWFA2) [1]. This remarkable cleavage specificity depends largely on the binding of the noncatalytic ADAMTS13 spacer domain to the C-terminal $\alpha$-helix of VWFA2 [2]. A 73 amino acid residue from D1596 to R1668 in VWF A2 domain, designated VWF73, serves as a minimal substrate for ADAMTS13 [3]. In concert, deletion of the VWFA2 C-terminal $\alpha$-helix (E1660-R1668) from this minimal substrate leads to nearly complete loss of cleavage by ADAMTS13, indicating that this structure is essential to the binding and cleavage of VWF by ADAMTS13 $[4,5]$.

We utilized standard hybridoma technology to develop monoclonal antibodies (mAbs) that detects the A2 domain of VWF (Additional file 1). One mAb (9G11), designated SZ-179, was identified as an immunoglobulin G1 (IgG1) subtype. SZ-179 interacts with

\footnotetext{
* Correspondence: ruanchanggeng@suda.edu.cn

${ }^{1}$ Jiangsu Institute of Hematology, Key Laboratory of Thrombosis and Hemostasis of Ministry of Health, The First Affiliated Hospital of Soochow University, Suzhou 215006, China

${ }^{2}$ Collaborative Innovation Center of Hematology, Soochow University,

Suzhou 215006, China
}

both the synthetic R1659-R1668 peptide (VWF 55 ) and native VWF with high affinity $(50 \mathrm{ng} / \mathrm{ml})$, as determined by enzyme-linked immunosorbent assay (ELISA) (Additional file 2: Figure S1). To identify the epitope of SZ-179, we evaluated the binding of this $\mathrm{mAb}$ to distinct VWF fragments, including VWFA1 (H-E1260P1467), VWFA2 (H-G1481R1668), VWFA3 (S1681R1877-H), and GSTVWF73-H (GST-D1596R1668-H) (Fig. 1a). As expected, SZ-179 bound to VWFA2 and GST-VWF73-H rather than VWFA1 or VWFA3 (Fig. 1b). These results suggest that SZ-179 mAb specifically targets the A2 domain WF. To further pinpoint the epitope recognized specifically bound to only VWFA2 (H-G1481R1668), VWFA2-C1 (H-G1481Q1667), -C2 (H-G1481L1666) and -N1(H-G1481P1658-E1660R1668) (Fig. 1d). These findings suggest that the epitope of SZ-179 is located within the distal portion of the VWFA2 domain between amino acid residues E1660-L1666.

Next, we determined if SZ-179 affects rADAMTS13mediated cleavage of the minimal substrate. We found that SZ-179, but not the isotype control murine IgG1, 
a

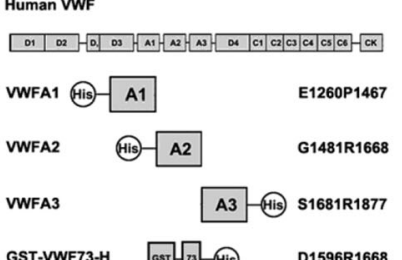

C

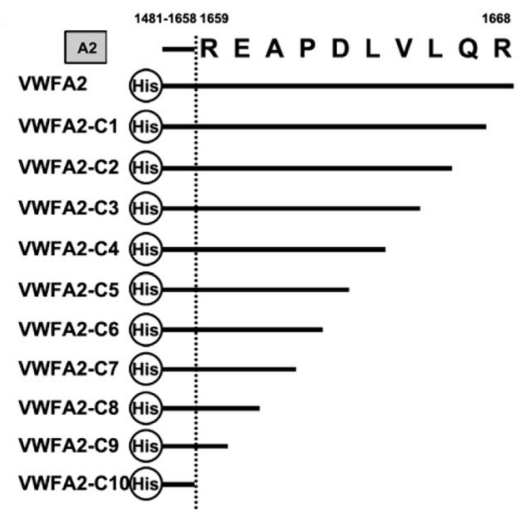

b
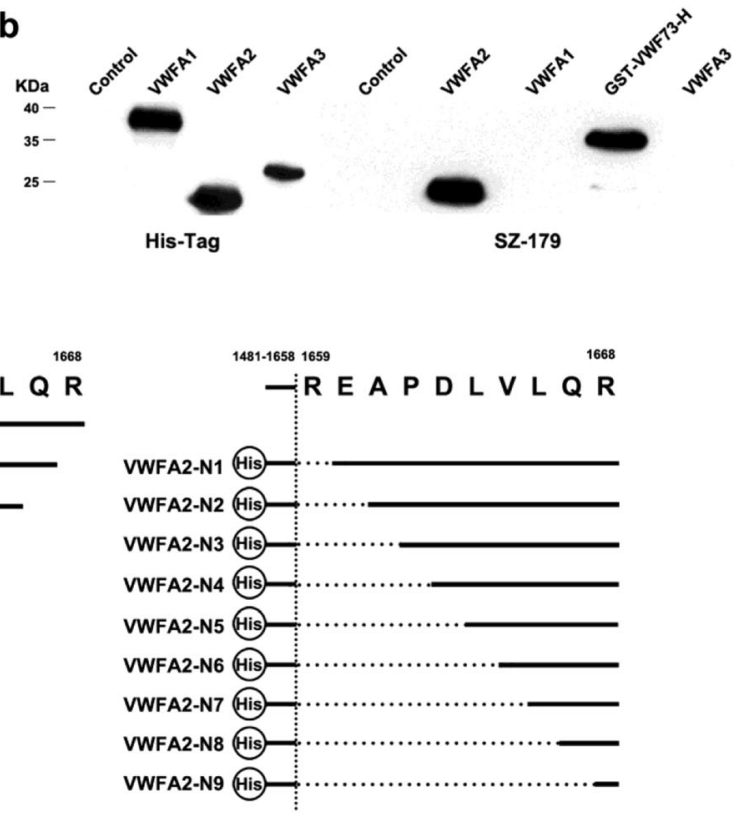

d

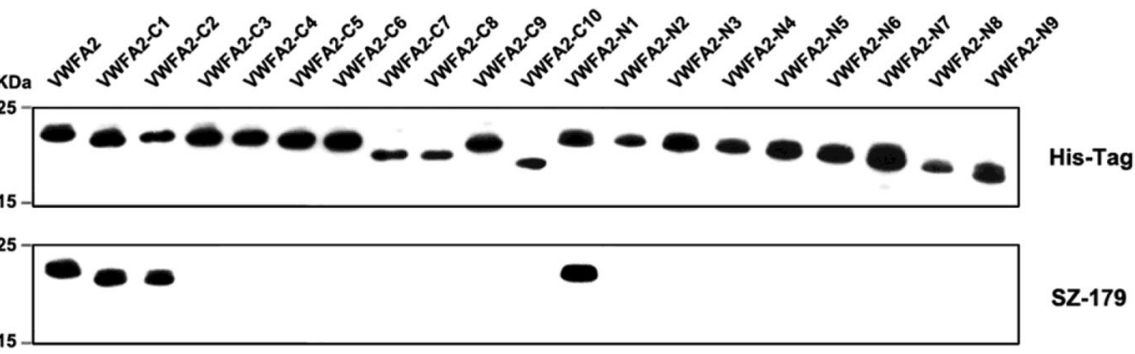

Fig. 1 Epitope mapping of SZ-179. a Schematic analysis of the human WWF. Domains are indicated. b WWFA1, WWFA2, GST-WWF73-H, and WWFA3 were separated by reduced 15\% SDS-PAGE and detected with a mouse anti-His antibody (left) or SZ-179 (right). c Schematic analysis of 20 different WWFA2 mutants with His tags. d WWFA2 and its deletion mutants were separated by 15\% reducing SDS-PAGE and detected by Western blotting with anti-His (top) or SZ-179 (bottom)

inhibited GST-VWF73-H cleavage by rADAMTS13 dose-dependently (Fig. 2a, b), with a half maximal inhibitory concentration (IC50) of $221.8 \mu \mathrm{g} / \mathrm{ml}$ (Additional file 3: Figure S2). In this light, SZ-179 abrogates cleavage of a minimal VWF substrate by rADAMTS13.

Moreover, we found that pre-incubation of plasma with SZ-179 rather than with the isotype control resulted in a dose-dependent decrease in the proteolysis of high molecular weight (HMW) VWF multimers under static/denaturing conditions, with an IC50 of $0.66 \mu \mathrm{g} / \mathrm{ml}$ (Additional file 3: Figure S2). These findings suggested that SZ-179 can bind to native VWF and provided further evidence that SZ-179 may attenuate the susceptibility of VWF to proteolytic cleavage by ADAMTS13 under physiological conditions.

We next determined whether SZ-179 could inhibit rADAMTS13-mediated proteolysis of the VWF-R1597W mutant, which can be cleaved by ADAMTS13 under static conditions and in the absence of denaturants including urea and guanidine [6, 7]. The R1597W mutation is commonly associated with von Willebrand disease (VWD) type 2A and located within VWFA2, close to the ADAMTS13 cleavage site. We found that the proteolysis of HMW VWF-R1597W multimers by rADAMTS13 was dramatically reduced by SZ-179 rather than by IgG1 isotype control in a concentrationdependent manner under native conditions (Fig. 2c, d). The IC50 of SZ-179 for this reaction was $13.54 \mu \mathrm{g} / \mathrm{ml}$ (Fig. 2e). Nevertheless, wild-type VWF treated with rADAMTS13 remained intact, as expected in the absence of chemical denaturation or fluid shear stress (Fig. 2f). These findings suggest that SZ-179 inhibits the rADAMTS13-mediated proteolysis of VWF-R1597W multimers under native conditions.

Mechanistically, SZ-179 may interact with E1660L1666 residues in the VWF, blocking the binding of the 
a

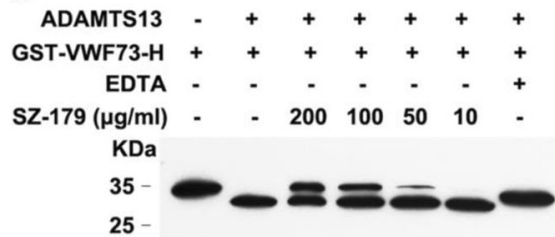

C

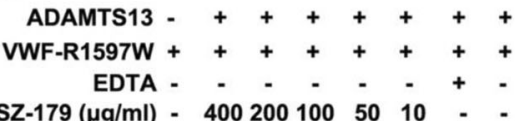

$\mathrm{SZ}-179(\mu \mathrm{g} / \mathrm{ml})-4002001005010 \quad-$

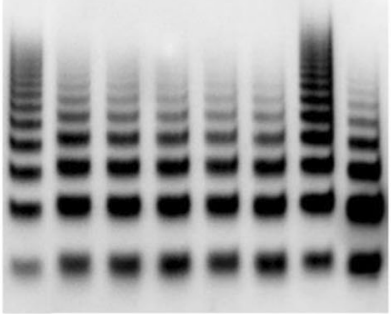

e

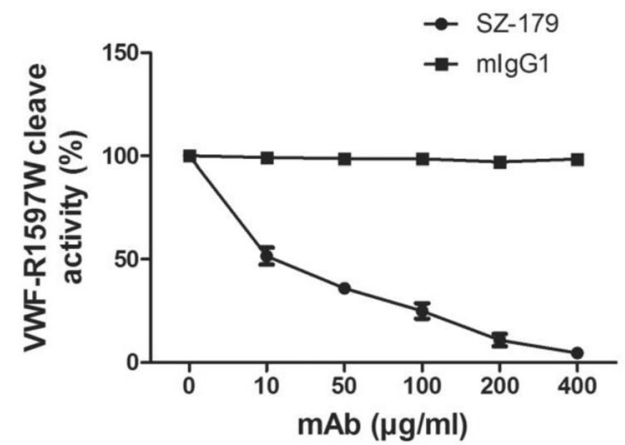

b

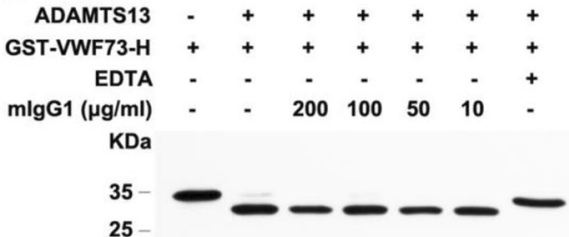

d

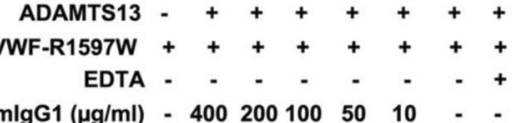

$\mathrm{mlgG1}(\mu \mathrm{g} / \mathrm{ml})-40020010050 \quad 10 \quad-$

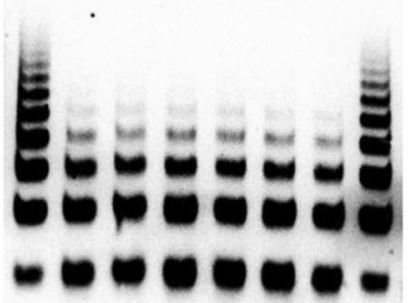

f

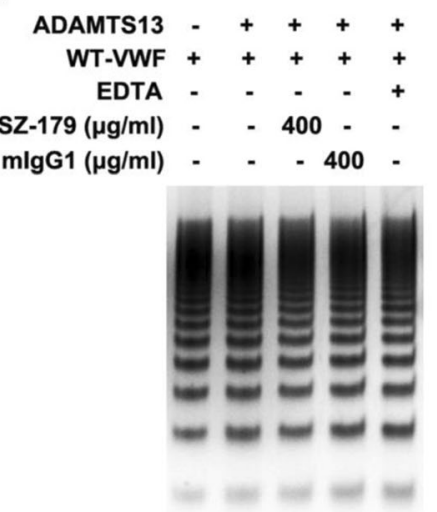

Fig. 2 SZ-179 inhibits rADAMTS13-mediated cleavage of the minimal substrate GSH-WWF73-H and WWF-R1597W under native conditions. a, b GST-VWF73-H $(2.8 \mu \mathrm{g})$ was pre-incubated with SZ-179 or isotype IgG1 for $2 \mathrm{~h}$ at $37^{\circ} \mathrm{C}$ and then incubated with $50 \mathrm{nM}$ rADAMTS13 for $1 \mathrm{~h}$. The cleavage products were analyzed by $15 \%$ reducing SDS-PAGE and Western blotting with an HRP-conjugated mouse anti-GST antibody. c, $\mathbf{d}$ VWFR1597W (150 nM) was incubated with SZ-179 or isotype control murine lgG1 at $4{ }^{\circ} \mathrm{C}$ for $30 \mathrm{~min}$, followed by $3 \mathrm{~h}$ with $50 \mathrm{nM}$ rADAMTS13 at $4{ }^{\circ} \mathrm{C}$. The proteolytic products were separated by electrophoresis in a $1.3 \%$ agarose gel and detected by anti-WWF. e Dose-response curve for inhibition of rADAMTS13-mediated cleavage of WWF-R1597W. f Wild-type WWF (150 nM) was treated with rADAMTS13 at $4{ }^{\circ} \mathrm{C}$ for $3 \mathrm{~h}$ as a control. Results represented as mean \pm SD of four independent experiments

spacer domain of ADAMTS13 to the substrate, thereby inhibiting proteolysis of VWF by ADAMTS13. Several recent reports support this possibility. For example, human neutrophil peptides inhibit ADAMTS13dependent VWF proteolysis by binding to the central A2 domain of VWF to block interactions between ADAMTS13 and VWF [8]. Antibody mAb508 is specific to the D4 domain of VWF, and has been observed to interfere with ADAMTS13-mediated degradation of
VWF in a vortex-based degradation assay [9]. mAb508 is bound to VWF with moderate affinity, and its binding to VWF partially inhibits the interaction between VWF and ADAMTS13. We discovered that SZ-179 has high affinity $(50 \mathrm{ng} / \mathrm{ml})$ with native VWF and prevents excessive degradation of HMW-VWF-multimers under denaturing conditions dose-dependently. SZ-179 may provide a promising therapeutic approach for a subset of VWD patients. 


\section{Additional files}

Additional file 1: Supplemental data. Detailed methods and materials are shown. (DOC $115 \mathrm{~kb}$ )

Additional file 2: Figure S1. Characterization of mAb SZ-179. (A) Quantification of ELISA analyses detecting SZ-179 binding to lgG1, IgG2a, IgG2a, lgG3, or lgM. (B) Quantification of ELISA analyses for SZ-179 or murine lgG1 binding to WFa5. Dose-response curves are shown. (C) Quantification of ELISA analyses for SZ-179 or murine lgG1 binding to plasma-derived WWF. Dose-response curves are shown. Data are mean \pm SD of four independent experiments. (DOCX $974 \mathrm{~kb})$

Additional file 3: Figure S2. SZ-179 inhibits cleavage of WF by ADAMTS13 in plasma under denaturing conditions. (A, B) Pooled normal human plasma was pre-incubated with SZ-179 or isotype control lgG1 for $2 \mathrm{~h}$ at $37^{\circ} \mathrm{C}$, and then incubated with $1.5 \mathrm{M}$ urea for $16 \mathrm{~h}$. The proteolytic products were separated by electrophoresis in a 1.3\% agarose gel and detected by anti-WWF. (C) Dose-response curve for inhibition of plasma ADAMTS13mediated cleavage of plasma-WWF. (D) Dose-response curve for inhibition of rADAMTS13-mediated GST-WWF73-H cleavage. Results represented as mean \pm SD of four independent experiments. (DOCX $1519 \mathrm{~kb}$ )

\section{Abbreviations}

ADAMTS13: A disintegrin and metalloproteinase with a thrombospondin type 1 motif, member 13; ELISA: Enzyme-linked immunosorbent assay; HMW: High molecular weight; IC50: Half maximal inhibitory concentration; IgG1: Immunoglobulin G1; mAb: Murine monoclonal antibody; WW: von Willebrand disease; WWF: von Willebrand factor

\section{Acknowledgements}

We acknowledge Blood Transfusion Hybridoma Laboratory Center for the gift of SP2/0-Ag14 myeloma cell line. Furthermore, we thank Dr. J. Evan Sadler for providing the PSVHVWF1 vector and Dr. Jingfei Dong for providing the pSecTag-ADAMTS13 vector.

\section{Funding}

This work was supported by Jiangsu Provincial Special Program of Medical Science (BL2012005), Jiangsu Province's Key Medical Center, National Natural Science Foundation of China (81600105) and the Priority Academic Program Development of Jiangsu Higher Education Institutions (PAPD).

\section{Availability of data and materials}

The dataset supporting the conclusions of this article is included within the article.

\section{Authors' contributions}

$L Z$ and $C R$ were the principal investigators and took primary responsibility for the paper. LZ, JS, FS, and ZM performed the experiments. YZ contributed the research material. LZ and LX wrote the paper. All authors read and approved the final manuscript.

\section{Competing interests}

The authors declare that they have no competing interests.

\section{Consent for publication}

Not applicable.

Ethics approval and consent to participate

Not applicable.

Received: 30 December 2016 Accepted: 25 January 2017 Published online: 06 February 2017

\section{References}

1. Gao W, Zhu J, Westfield LA, Tuley EA, Anderson PJ, Sadler JE. Rearranging exosites in noncatalytic domains can redirect the substrate specificity of ADAMTS proteases. J Biol Chem. 2012;287:26944-52.

2. Crawley JT, de Groot R, Xiang Y, Luken BM, Lane DA. Unraveling the scissile bond: how ADAMTS13 recognizes and cleaves von Willebrand factor. Blood. 2011;118:3212-21.
3. Kokame K, Matsumoto M, Fujimura Y, Miyata T. WWF73, a region from D1596 to R1668 of von Willebrand factor, provides a minimal substrate for ADAMTS-13. Blood. 2004;103:607-12.

4. Wu JJ, Fujikawa K, McMullen BA, Chung DW. Characterization of a core binding site for ADAMTS-13 in the A2 domain of von Willebrand factor. Proc Natl Acad Sci U S A. 2006;103:18470-4.

5. Gao W, Anderson PJ, Sadler JE. Extensive contacts between ADAMTS13 exosites and von Willebrand factor domain A2 contribute to substrate specificity. Blood. 2008;112:1713-9.

6. Hassenpflug WA, Budde U, Obser T, Angerhaus D, Drewke E, Schneppenheim S, et al. Impact of mutations in the von Willebrand factor A2 domain on ADAMTS13-dependent proteolysis. Blood. 2006;107:2339-45.

7. Xu AJ, Springer TA. Mechanisms by which von Willebrand disease mutations destabilize the A2 domain. J Biol Chem. 2013;288:6317-24.

8. Pillai VG, Bao J, Zander CB, McDaniel JK, Chetty PS, Seeholzer SH, et al. Human neutrophil peptides inhibit cleavage of von Willebrand factor by ADAMTS13: a potential link of inflammation to TTP. Blood. 2016:128:110-9.

9. Rauch A, Legendre P, Christophe OD, Goudemand J, van Belle E, Vincentelli A, et al. Antibody-based prevention of von Willebrand factor degradation mediated by circulatory assist devices. Thromb Haemost. 2014;112:1014-23.

\section{Submit your next manuscript to BioMed Central and we will help you at every step:}

- We accept pre-submission inquiries

- Our selector tool helps you to find the most relevant journal

- We provide round the clock customer support

- Convenient online submission

- Thorough peer review

- Inclusion in PubMed and all major indexing services

- Maximum visibility for your research

Submit your manuscript at www.biomedcentral.com/submit
Biomed Central 